\title{
Serum galectin-3 and galectin-3 binding protein levels in systemic lupus erythematosus and cutaneous lupus erythematosus
}

\author{
Agnieszka Kalinska-Bienias ${ }^{1}$, Emilia Kowalczyk ${ }^{1}$, Piotr Bienias², Kamila Gala³, Pawel Jagielski ${ }^{4}$, Cezary Kowalewski ${ }^{1}$ \\ 1Department of Dermatology and Immunodermatology, Medical University of Warsaw, Warsaw, Poland \\ 2Department of Internal Medicine and Cardiology, Medical University of Warsaw, Warsaw, Poland \\ ${ }^{3}$ Department of Immunology, Transplantology and Internal Diseases, Medical University of Warsaw, Warsaw, Poland \\ ${ }^{4}$ Human Nutrition Department Faculty of Health Science, Jagiellonian University Medical College, Krakow, Poland \\ Adv Dermatol Allergol 2021; XXXVIII (2): 274-280 \\ DOI: https://doi.org/10.5114/ada.2020.92320
}

\begin{abstract}
Introduction: The roles of galectin-3 (Gal-3) and galectin-3 binding protein (G3BP) in systemic lupus erythematosus (SLE) are of ongoing interest, but the data are insufficient due to highly limited available studies. There are no data on cutaneous lupus erythematosus (CLE).

Aim: To assess serum Gal-3 and G3BP concentrations in SLE patients with and without LE-specific skin lesions, CLE patients and to correlate levels of proteins with clinical and laboratory parameters.

Material and methods: The study included 71 SLE patients with and without LE-specific skin lesions, 23 CLE patients and 40 controls. Gal-3 and G3BP were measured by specific enzyme-linked immunosorbent assays (ELISA).

Results: Serum Gal-3 and G3BP concentrations were significantly higher in SLE with and without LE-specific lesions compared to controls, but without differences between SLE groups. Gal-3 and G3BP levels were also elevated in CLE compared to controls ( $p=0.001, p=0.005$; respectively). There was a positive correlation between G3BP level and CLASI activity score both in CLE $(r=0.55, p=0.006)$ and in SLE patients with LE-specific lesions $(r=0.36$, $p=0.02)$. G3BP and Gal-3 levels did not differ in SLE with LE-specific lesions and CLE. There was a positive correlation between serum G3BP level and the SLEDAI score in SLE patients $(r=0.26, p=0.03)$.

Conclusions: Our findings indicate that serum G3BP and Gal-3 are elevated in CLE. Additionally, G3BP might be associated with the extent of skin lesions. There are no differences between G3BP and Gal-3 concentrations in SLE with and without LE-specific skin lesions.
\end{abstract}

Key words: galectin-3, galectin-3 binding protein, lupus erythematosus.

\section{Introduction}

Lupus erythematosus (LE) is an inflammatory autoimmune disease, which encompasses multiple systemic organ involvement (systemic lupus erythematosus - SLE), but frequently affects only the skin (cutaneous lupus erythematosus - CLE). Both diseases are related to each other as approximately $12 \%$ of patients are first diagnosed as only skin affected CLE progresses to SLE [1]. Furthermore, CLE develops in $72-85 \%$ of SLE patients in the course of disease; thus skin is the second most commonly affected organ in SLE (followed by arthritis) [2]. Four subtypes of CLE were defined: (i) acute (ACLE); (ii) subacute (SCLE); (iii) chronic (CCLE), including discoid LE (DLE), chilblain
LE (CHLE) and LE panniculitis (LEP); and (iv) intermittent (ICLE), synonymously LE tumidus (LET) [3].

The etiology of SLE and CLE remains unclear despite ongoing research. Potential biomarkers which in recent years have been of particular scientific interest and may contribute to the role in the pathogenesis of LE are galectin-3 (Gal-3) and its binding protein (galectin-3 binding protein - G3BP). However, current knowledge on this subject is incomplete and based on a few research results.

Galectin-3, also named as macrophage surface marker (Mac-2) or carbohydrate binding protein 35, belongs to the family of lectins that bind $\beta$-galactosides. Up

Address for correspondence: Agnieszka Kalinska-Bienias MD, PhD Department of Dermatology and Immunodermatology, Medical University of Warsaw, 82 a Koszykowa St, 02-008 Warsaw, Poland, phone: +48 606618 564, fax: +48 50221 04, e-mail: agnieszka.kalinska@interia.pl. Received: 23.10.2019, accepted: 4.11.2019. 
to now at least 15 galectins have been identified. Gal3 plays an extracellular role, inducing the apoptosis of T-cells and monocytes, and an intracellular one with its anti-apoptotic activity [4, 5]. The expression of Gal-3 is stimulated by the hypomethylation of the gene promoter. Consequently, DNA hypomethylation is seen in various connective tissue diseases, including SLE [6].

Originally described as a macrophage surface marker binding protein (Mac-2 BP), G3BP is a scavenger cysteinerich domain receptor that exerts its activity by binding to lectins, integrins and extracellular matrix proteins. G3BP is a ligand for galectin-3 and an important factor of cell clearance, apoptosis, intercellular adhesion and signaling [7].

Data on the serum concentration of Gal-3 and G3BP in SLE were initially derived from the study of patients with Behcet's disease in which SLE patients represented a control group. Interestingly, serum Gal-3 and G3BP proteins were much higher in SLE than in Behcet's disease and healthy groups [8]. The results of further studies revealed that Gal-3 and G3BP concentrations were elevated in the sera of SLE patients compared to healthy controls [9-14]. However, by reason of the pioneering character of the research as well as the small number of the study groups, their role as markers of the immune and inflammatory processes in SLE requires further investigation. In a particularly interesting study, Shi et al. showed that the expression of Gal-3 in skin biopsies obtained from LE-specific skin lesions was reduced. In addition, the authors compared serum Gal-3 data in patients with SLE with and without LE skin lesions and found no difference between the groups [9]. It indicates that the role of Gal-3 in LE skin lesions needs to be elucidated. To the best of our knowledge, there are no data on Gal-3 and G3BP in patients with CLE as well as no evaluation of G3BP level in SLE with and without LE-specific skin lesions.

\section{Aim}

The aim of this study was to assess the serum Gal-3 and G3BP concentrations in SLE patients (with and without LE-specific lesions) and in CLE patients compared to healthy subjects and to correlate the concentration of those proteins with clinical and laboratory features.

\section{Material and methods}

\section{Patients}

The study included 71 patients diagnosed with SLE, 23 patients with CLE and 40 healthy subjects as a control group. The diagnosis of SLE was determined according to the American College of Rheumatology (ACR) revised criteria if the patients fulfilled four or more than four [15]. Patients with SLE were classified into two groups based on the presence of LE-specific skin lesions at the time of serum collections: (i) SLE with LE-specific skin lesions
(38 patients), (ii) SLE without LE-specific skin lesions (33 patients). The LE-specific skin lesions presented ACLE, SCLE and/or CCLE. In some patients common skin manifestations, e.g. livedo reticularis, Raynaud's phenomenon, or periungual telangiectasia, were found, but these were not classified as LE-specific skin manifestations. The SLE activity was assessed by the Systemic Lupus Erythematosus Disease Activity Index (SLEDAI) [16]. The diagnosis in patients with CLE was determined on the basis of clinical features, duration of skin lesions and histopathological study of skin lesions. All CLE patients manifested skin lesions at the time of serum collections and presented less than four ACR criteria. Additionally, immunofluorescent study of biopsies (lupus band test) taken from skin lesions was performed in $85 \%$ of CLE patients. The extent of skin lesions in patients with SLE and CLE was assessed by the Cutaneous Lupus erythematosus disease Area and Severity Index (CLASI) activity and damage scores [17]. Routine laboratory evaluation of white blood cell count $(\mathrm{WBC})$, red blood count $(\mathrm{RBC})$, platelet count $(\mathrm{PLT})$, erythrocyte sedimentation rate (ESR), C-reactive protein (CRP) and complement 3 (C3) and 4 (C4) was assessed by standard laboratory methods. Indirect immunofluorescence on human Hep-2 cells was used to determine antinuclear antibodies (ANA). Double-stranded DNA antibodies were assessed on Crithidia luciliae. Specification of ANA was performed by immunoblot technique and/or the immunodiffusion method. Consent of the local Bioethics Committee was obtained prior to the study. All patients and controls gave informed consent and institutional approval was obtained.

\section{Measurement of serum galectin-3 and galectin-3 binding protein concentrations}

Serum samples were stored at $-20^{\circ} \mathrm{C}$ until assessment. Specific enzyme-linked immunosorbent assay (ELISA) kits were used to measure serum Gal-3 (Wuhan Fine Biological Technology Co., Ltd., Wuhan, China) and G3BP concentrations (human 90K/Mac-2BP platinum ELISA kit, Bender MedSystems, Vienna, Austria) in patients and the control group. The procedures were performed according to the manufacturer's instructions. Serum Gal-3 and G3BP concentrations were calculated using the standard curve. All experiments were carried out in duplicate.

\section{Statistical analysis}

All statistical analyses were performed using the statistical software packages IMAGO PRO Academic (IBM SPSS Statistics 25) and SAS Enterprise Guide 7.1. Variables with a normal distribution are presented as mean followed by standard deviation (SD). For categorical variables, the differences between groups were compared by the $\chi^{2}$ test. Each parameter was assessed for normality with the Shapiro-Wilk test. The Mann-Whitney- $U$ test was used for the comparison of non-parametric continu- 
ous variables between two groups. An analysis of variance (ANOVA) or Kruskal-Wallis was used to compare the values between three groups. A post-hoc analysis was conducted using Tukey's significance test. The correlation analyses were performed by Spearman's rank correlation test. The value of $p<0.05$ was considered significant.

\section{Results}

The characteristics of SLE patients with and without LE-specific skin lesions and CLE patients are shown in Table 1. The mean age of SLE patients with and without LE-specific skin lesions, CLE patients and the control group was $48.0 \pm 16.4,54.0 \pm 14.3,52.5 \pm 14.4$ and 48.4 \pm 12.9 years, respectively. There was no difference in disease duration between studied groups. Arthritis, kidney disease and neurological diseases occurred with similar frequency in groups of SLE patients. The median SLEDA score was higher in SLE patients with LE-specific skin lesions than without LE-specific skin lesions ( $p=0.02)$, mainly due to the presence of skin lesions. The median CLASI activity score was higher in SLE patients with LEspecific skin lesions than in CLE patients $(p=0.02)$ while the CLASI damage score was similar $(p=0.77)$.

\section{Serum galectin-3 and galectin-3 binding protein concentrations}

The mean serum Gal-3 concentration was 10.46 $\pm 3.25 \mathrm{ng} / \mathrm{ml}$ in SLE with LE-specific skin lesions, 10.69 $\pm 3.42 \mathrm{ng} / \mathrm{ml}$ in SLE without LE-specific skin lesions, 10.15 \pm 2.68 in CLE, $6.92 \pm 3.47 \mathrm{ng} / \mathrm{ml}$ in the control group (Figure $1 \mathrm{~A})$. The mean concentration of serum Gal-3 did not differ between CLE and SLE with LE-skin lesions ( $p=1.0)$. Similarly, there was no difference between SLE with LE-specific skin lesions vs. SLE without LE-specific skin lesions $(p=1.0)$. The mean serum G3BP concentration was $13714.7 \pm 4595.5 \mu \mathrm{g} / \mathrm{ml}$ in SLE with LE-specific skin lesions, $11399.6 \pm 3760.9 \mu \mathrm{g} / \mathrm{ml}$ in SLE without LE-specific skin lesions, $10964.0 \pm 3866.6 \mu \mathrm{g} / \mathrm{ml}$ in CLE and 7870.0 $\pm 3113.8 \mu \mathrm{g} / \mathrm{ml}$ in the control group. The concentrations of G3BP were significantly higher in all groups of patients compared to the control group (Figure $1 \mathrm{~B}$ ). The level of G3BP did not differ in CLE and SLE with LE-specific skin lesions. No difference in serum G3BP concentration was found between SLE patients with and without LE-skin lesions $(p=0.28)$.

\section{Correlations of serum galectin-3 and galectin-3 binding protein concentrations with clinical and laboratory parameters}

A positive correlation was observed between serum G3BP concentration and CLASI activity score in CLE ( $r=$ $0.55, p=0.006$ ) (Figure $2 \mathrm{~A}$ ). Serum G3BP level also correlated with CLASI activity score in SLE with LE-specific skin lesions $(r=0.36, p=0.02$ ) (Figure $2 \mathrm{~B}$ ). There was a posi- tive correlation between serum G3BP concentration and the SLEDAI score in SLE patients $(r=0.26, p=0.03$ ) (Figure $2 \mathrm{C}$ ). A positive correlation was observed between serum G3BP concentration and ESR in SLE patients $(r=0.3$, $p=0.01$ ). Serum G3BP concentration did not correlate with other clinical and laboratory parameters (disease duration, CLASI damage score, serum levels of C3 and C4, ANA, arthritis, kidney and neurological diseases). No correlations were observed between serum Gal-3 concentration and disease duration, the SLEDAI score, ESR, levels of C3 and C4, ANA, arthritis, kidney and neurological diseases (precise data not shown).

\section{Discussion}

The diversity of functions performed by Gal-3 and G3BP causes an increasing interest in those proteins in the context of inflammatory and autoimmune disorders. It seems that they could contribute to a fuller understanding of lupus erythematosus pathogenesis. Available scientific reports indicate an increased serum level of Gal-3 in patients with SLE [9-11, 14, 18]. As regards G3BP, a particularly promising assessment was presented by the Nielsen et al. study (2015), in which the authors observed a higher serum G3BP concentration in SLE patients, especially in lupus nephritis [19]. Our study also showed that serum Gal-3 and G3BP concentrations were significantly higher in SLE patients.

To the best of our knowledge, we are the first to assess Gal-3 and G3BP serum concentrations in CLE patients, noting the increased levels of both proteins in this disease. Unexpectedly, the concentration of Gal-3 and G3BP was similar in patients with CLE and SLE with LE-specific skin lesions. The reason for these results is difficult to explain as CLE remains mainly a skin problem, not a systemic one. Nevertheless, some disturbances typical of systemic involvement are observed in CLE, but they are not sufficient to meet the criteria necessary for the diagnosis of SLE. We hypothesized that the high concentration of Gal-3 and G3BP in CLE may be associated with the lack of immunosuppressive therapy (usually systemic steroids) which was applied in most SLE patients as opposed to patients with CLE.

Our results suggest that elevated serum G3BP concentration may reflect the extent of skin lesions. We did not make such an observation in relation to Gal-3, which is consistent with the results of the Shi et al. study from 2018. On the other hand, there is evidence that Gal-3 may contribute to the development of lupus skin lesions by recruiting inflammatory cells for the dermis. The analysis of lupus skin biopsies in patients with SLE revealed that Gal-3 expression was reduced in the epidermis of LE-specific skin lesions compared to healthy controls (lower in chronic lesions than in acute ones) [9]. It is suggested that a decrease in Gal-3 expression in the epidermis may be associated with its involvement in lymphoid 
Table 1. Clinical and laboratory characteristics of patients with systemic lupus erythematosus with lupus-specific skin lesions, systemic lupus erythematosus without lupus-specific skin lesions and discoid lupus erythematosus (NA - not available). P-value in one row shows statistical differences between 2 or 3 values depending on characteristic

\begin{tabular}{|c|c|c|c|c|}
\hline Parameter & $\begin{array}{l}\text { SLE with skin lesions } \\
\quad(n=38)\end{array}$ & $\begin{array}{l}\text { SLE without skin lesions } \\
\quad(n=33)\end{array}$ & CLE $(n=23)$ & $P$-value \\
\hline \multicolumn{5}{|l|}{ Clinical data: } \\
\hline Age at enrolment [years] & $48.0 \pm 16.4$ & $54.0 \pm 14.3$ & $52.5 \pm 14.4$ & 0.22 \\
\hline Age at onset [years] & $38.8 \pm 18.7$ & $38.6 \pm 13.8$ & $38.9 \pm 19.6$ & 0.99 \\
\hline Gender, $n$ male/female & $5 / 33$ & $1 / 32$ & $6 / 17$ & 0.04 \\
\hline Disease duration, (range) [years] & $6.5(1-35)$ & $16(1-30)$ & $7(0-44)$ & 0.07 \\
\hline Arthritis, $n(\%)$ & $29(76.3)$ & $24(72.7)$ & - & 0.96 \\
\hline Kidney disease, $n(\%)$ & $10(26.3)$ & $8(24.2)$ & - & 0.84 \\
\hline Neurological diseases, $n(\%)$ & $8(21.1)$ & $7(21.2)$ & - & 0.98 \\
\hline CLASI activity score (range) & $8(2-20)$ & - & $4(2-21)$ & 0.02 \\
\hline CLASI damage score (range) & $3(0-10)$ & - & $3(0-23)$ & 0.77 \\
\hline SLEDAI score (range) & $8(2-18)$ & $6(0-22)$ & - & 0.02 \\
\hline \multicolumn{5}{|l|}{ Laboratory and immunologic data: } \\
\hline $\mathrm{WBC}\left[\times 10^{3} / \mathrm{mm}^{3}\right]$ & $5.8 \pm 2.7$ & $5.8 \pm 2.0$ & $6.1 \pm 1.5$ & 0.82 \\
\hline $\mathrm{RBC}\left[\times 10^{9} / 1\right]$ & $4.4 \pm 0.5$ & $4.4 \pm 0.3$ & $4.6 \pm 0.5$ & 0.19 \\
\hline $\mathrm{PLT}\left[\times 10^{3} / \mathrm{mm}^{3}\right]$ & $227.0 \pm 96.0$ & $215.0 \pm 83.6$ & $228.7 \pm 64.8$ & 0.41 \\
\hline $\mathrm{ESR}[\mathrm{mm} / \mathrm{h}]$ & $12.5(3-86)$ & $18(4-59)$ & $11.7 \pm 11.1$ & 0.02 \\
\hline CRP & $1.6(0.2-30)$ & $2(0.3-51)$ & $1.4(0.1-10)$ & 0.29 \\
\hline ANA mean titer \pm SD & $480(80-5120)$ & $320(80-2560)$ & $160(0-2560)$ & $<0.0001$ \\
\hline Anti-ds-DNA, $n(\%)$ & $8(21.1)$ & $5(15.2)$ & $1(4.3)$ & 0.21 \\
\hline Anti-Ro, $n$ (\%) & $19(50.0)$ & $17(51.5)$ & $0(0)$ & 0.0001 \\
\hline Anti-La, $n$ (\%) & $5(13.2)$ & $5(15.2)$ & $0(0)$ & 0.16 \\
\hline Anti-RNP, $n(\%)$ & $5(13.2)$ & $3(9.1)$ & $1(4.3)$ & 0.52 \\
\hline Anti-Sm, $n(\%)$ & $2(5.3)$ & $1(3.0)$ & $0(0)$ & 0.52 \\
\hline Anti-histone, $n(\%)$ & $1(2.6)$ & $0(0)$ & $0(0)$ & 0.47 \\
\hline C3 complement [mg/l] & $97.7 \pm 33.4$ & $99.8 \pm 29.2$ & $110.2 \pm 15.3$ & 0.13 \\
\hline C4 complement [mg/l] & $18.4 \pm 10.8$ & $18.1 \pm 9.9$ & $25.4 \pm 11.7$ & 0.03 \\
\hline \multicolumn{5}{|l|}{ Treatment, $n$ (\%): } \\
\hline Glucocorticosteroids & $33(86.8)$ & $23(69.7)$ & $2(8.7)$ & $<0.0001$ \\
\hline Antimalarial drugs & $19(50.0)$ & $16(48.5)$ & $18(78.3)$ & 0.05 \\
\hline Cyclosporine & $5(13.2)$ & $0(0)$ & $0(0)$ & 0.02 \\
\hline Methotrexate & $1(2.6)$ & $4(12.1)$ & $0(0)$ & 0.09 \\
\hline Cyclophosphamide & $0(0)$ & $1(3.0)$ & $0(0)$ & 0.39 \\
\hline Mycophenolate mofetil & $3(7.9)$ & $1(3.0)$ & $0(0)$ & 0.30 \\
\hline Azathioprine & $7(18.4)$ & $7(21.2)$ & $1(4.3)$ & 0.21 \\
\hline Dapsone & $3(7.9)$ & $0(0)$ & $0(0)$ & 0.10 \\
\hline
\end{tabular}

infiltrates in the dermis as Gal-3 is known to activate many lymphoid cells and act as a chemoattractant for human monocytes and macrophages. Moreover, in vivo studies using Gal-3 deficient mice support the notion that Gal-3 promotes inflammatory responses in mouse models [20]. It has also been shown that Gal-3 knock- 

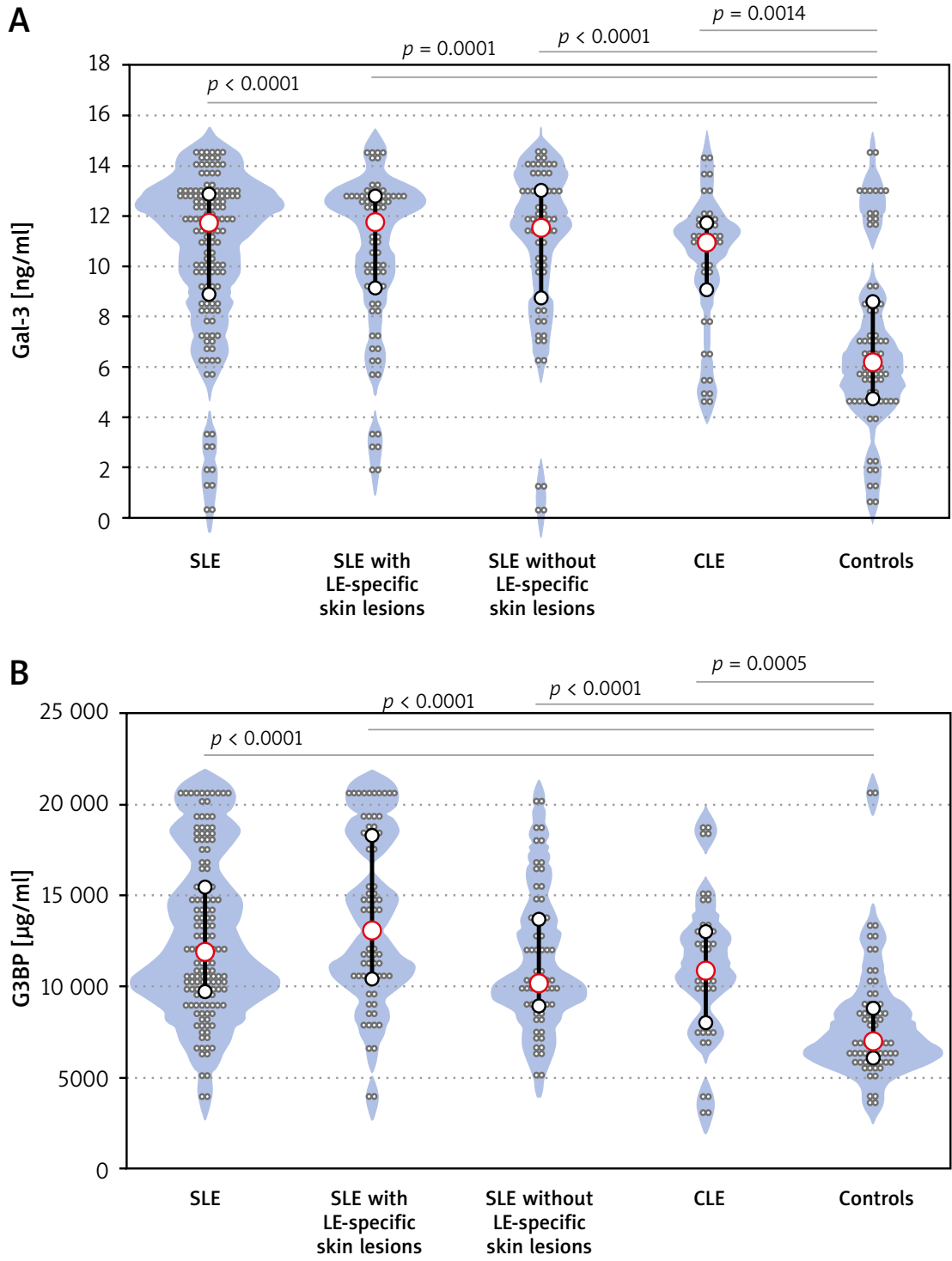

Figure 1. A - Serum galectin-3 (Gal-3) concentration in systemic lupus erythematosus (SLE), SLE with LE-specific skin lesions, SLE without LE-specific skin lesions, cutaneous lupus erythematosus (CLE), B - control group serum galectin-3 binding protein (G3BP) concentration in SLE, SLE with LE-specific skin lesions, SLE without LE-specific skin lesions, CLE and control group

down resulted in the overexpression of chemokines such as CXCL1 and CCL20, and enhanced the peripheral blood mononuclear cell-attracting capacity of keratinocytes [9]. It is worth mentioning that purified anti-Gal-3 antibodies administered subcutaneously to mice resulted in LE histopathological changes (vasculitis and epidermal atrophy) [21]. Interestingly, in the present study we found that in patients with SLE there was no difference in Gal3 concentration in groups with and without LE-specific skin lesions. Similar results were obtained by Shi et al. in 2018 [9]. It is noteworthy that in the present study we noted for the first time that patients with SLE with and without skin lesions did not differ in G3BP levels. These results suggest that the presence of skin lesions in SLE patients probably does not affect both Gal-3 and G3BP concentrations.

Nevertheless, available literature provides data on Gal-3 and G3BP as novel biomarkers of SLE activity [811]. Our analysis also supported a positive correlation between G3BP concentration and SLEDAI score in SLE patients; however, we did not find a correlation between Gal-3 and SLEDAI score. It is worth mentioning that SLE patients had much higher levels of Gal-3 and G3BP compared to other connective tissue diseases such as rheumatoid arthritis, Behcet's disease or systemic sclerosis, although the concentrations of Gal-3 and G3BP were found to be elevated in those diseases [10]. It should also be underlined that the concentration of Gal-3 was 
A

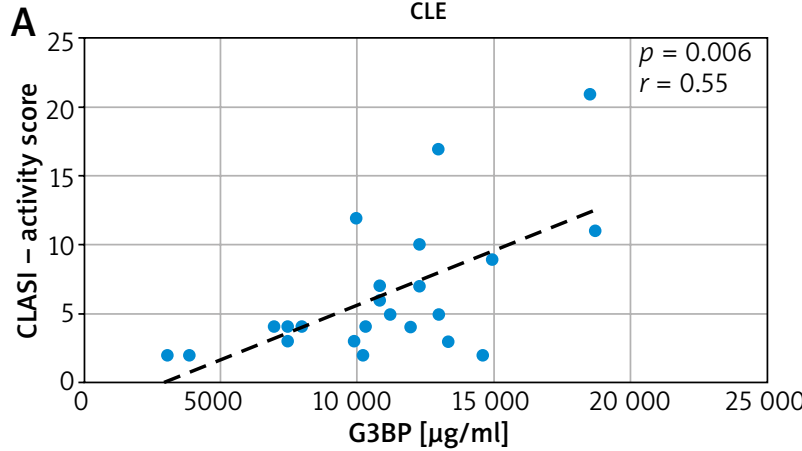

C

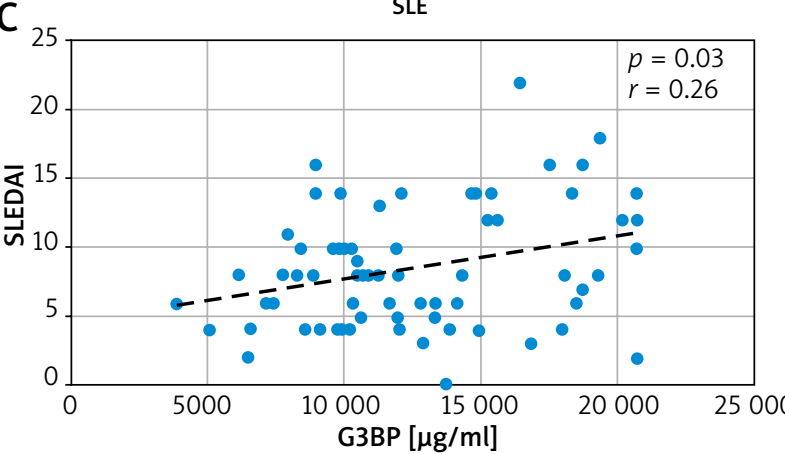

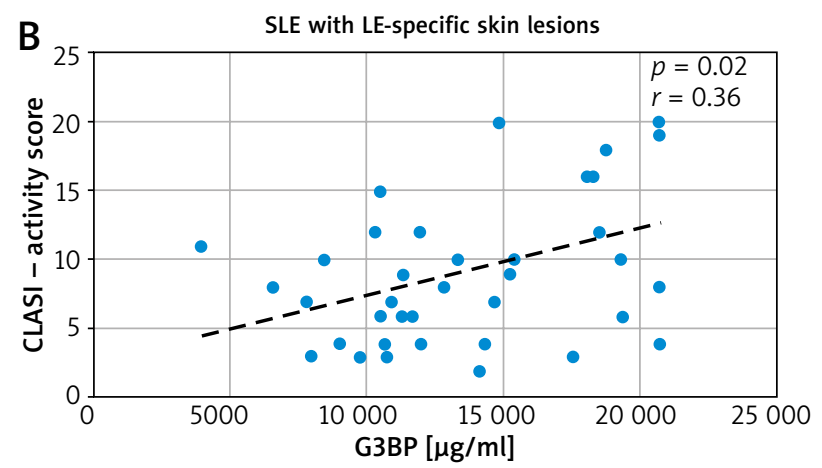

Figure 2. Correlation between serum galectin-3 binding protein (G3BP) concentration: A - Cutaneous Lupus erythematosus disease Area and Severity Index (CLASI) activity score in cutaneous lupus erythematosus (CLE), B - CLASI activity score in systemic lupus erythematosus (SLE) with LE-specific skin lesions, C - Systemic Lupus Erythematosus Disease Activity Index (SLEDAI) in systemic lupus erythematosus (SLE) patients revealed to be higher in SLE than in systemic sclerosis, although Gal-3 is widely considered as a marker of fibrosis, providing extensive proliferation of fibroblasts and stimulation of the synthesis of collagen (mainly type I) [10, 22].

The strict mechanism of Gal-3 and G3BP function in SLE is not clearly understood. It is known that typical for SLE pathogenesis is the defective clearance of apoptotic cells. Galectins are used as agents whose task is to recognize apoptotic cells and then through the glycosylation process to change their structure so that they are recognized by the immune system [5]. G3BP was found as their distinguishable biomarker.

Some data confirm that micro-particles derived from cellular apoptosis are the source of autoantigens and Gal-3 or G3BP may be among them [21]. Kang et al. analyzing renal biopsies in patients with lupus nephritis found significantly elevated glomerular expression of Gal-3 in renal tissue [11]. Regarding G3BP, it has been shown by Nielsen et al. that G3BP was present together with immune complexes in kidney tissues in SLE patients with renal involvement [19].

In addition, it was found that G3BP plasma level mirrors type I interferon (IFN) activity [13]. Increased G3BP activity is also present in $\alpha$-IFN-activated neutrophils and peripheral blood mononuclear cells in SLE patients. Increased activity of IFN and G3BP in connection with an excess of circulating molecules containing fragments of apoptotic cells may be a factor triggering such a strong, systemic autoimmune reaction in SLE [7].
The practical value of Gal-3 and G3BP evaluation is an open issue. Due to numerous connections between Gal-3, G3BP and lupus erythematosus, it seems that further research will facilitate considering them as disease biomarkers in routine examinations. Moreover, some scientific reports have demonstrated that galectins have a great deal of therapeutic potential. We hope that our findings might be a proper direction for the development of galectin-based therapeutic agents.

The limitation of this study is the small representation of the patients resulting from the assessment carried out by a single university center.

\section{Acknowledgments}

Thank you to Professor B. Foroncewicz from the Faculty of Immunology, Transplantology and Internal Diseases, Warsaw Medical University in Poland for substantive support of this study and help in recruiting patients. We also thank Dr M. Słowik-Rylska from the Medical Faculty of the Jan Kochanowski University in Kielce for participating in collecting a group of patients.

\section{Conflict of interest}

The authors declare no conflict of interest.

\section{References}

1. Schultz HY, Dutz JP, Furukawa F, et al. From pathogenesis, epidemiology, and genetics to definitions, diagnosis, and 
treatments of cutaneous lupus erythematosus and dermatomyositis: a report from the 3rd International Conference on Cutaneous Lupus Erythematosus (ICCLE) 2013. J Invest Dermatol 2015; 135: 7-12.

2. Stannard JN, Kahlenberg JM. Cutaneous lupus erythematosus: updates on pathogenesis and associations with systemic lupus. Curr Opin Rheumatol 2016; 28: 453-9.

3. Kuhn A, Landmann A. The classification and diagnosis of cutaneous lupus erythematosus. J Autoimmun 2014; 48 49: 14-9.

4. Dumic J, Dabelic S, Flogel M. Galectin-3: an open-ended story. Biochim Biophys Acta 2006; 1760: 616-35.

5. Yang RY, Rabinovich GA, Liu FT. Galectins: structure, function and therapeutic potential. Expert Rev Mol Med 2008; 10: e17.

6. Ohshima S, Kuchen S, Seemayer CA, et al. Galectin 3 and its binding protein in rheumatoid arthritis. Arthritis Rheum 2003; 48: 2788-95.

7. Nielsen CT, Ostergaard O, Rasmussen NS, et al. A review of studies of the proteomes of circulating microparticles: key roles for galectin-3-binding protein-expressing microparticles in vascular diseases and systemic lupus erythematosus. Clin Proteomics 2017; 14: 11.

8. Lee YJ, Kang SW, Song JK, et al. Serum galectin-3 and galectin-3 binding protein levels in Behcet's disease and their association with disease activity. Clin Exp Rheumatol 2007; 25: S41-5.

9. Shi Z, Meng Z, Han Y, et al. The involvement of galectin-3 in skin injury in systemic lupus erythematosus patients. Lupus 2018; 27: 621-7.

10. Koca SS, Akbas F, Ozgen M, et al. Serum galectin-3 level in systemic sclerosis. Clin Rheumatol 2014; 33: 215-20.

11. Kang EH, Moon KC, Lee EY, et al. Renal expression of galectin-3 in systemic lupus erythematosus patients with nephritis. Lupus 2009; 18: 22-8.

12. Ostergaard O, Nielsen CT, Iversen LV, et al. Unique protein signature of circulating microparticles in systemic lupus erythematosus. Arthritis Rheum 2013; 65: 2680-90.

13. Nielsen CT, Lood C, Ostergaard O, et al. Plasma levels of galectin-3-binding protein reflect type I interferon activity and are increased in patients with systemic lupus erythematosus. Lupus Sci Med 2014; 1: e000026.

14. Lim Y, Lee DY, Lee S, et al. Identification of autoantibodies associated with systemic lupus erythematosus. Biochem Biophys Res Commun 2002; 295: 119-24.

15. Hochberg MC. Updating the American College of Rheumatology revised criteria for the classification of systemic lupus erythematosus. Arthritis Rheum 1997; 40: 1725.

16. Bombardier C, Gladman DD, Urowitz MB, et al. Derivation of the SLEDAI. A disease activity index for lupus patients. The Committee on Prognosis Studies in SLE. Arthritis Rheum 1992; 35: 630-40.

17. Albrecht J, Werth VP. Development of the CLASI as an outcome instrument for cutaneous lupus erythematosus. Dermatol Ther 2007; 20: 93-101.

18. Kalińska-Bienias A, Foroncewicz B, Bienias P, Kowalewski C. Selected novel aspects in pathogenesis of lupus erythematosus - interdisciplinary view. Dermatol Rev 2016; 103: 345-53.

19. Nielsen CT, Ostergaard O, Rekvig OP, et al. Galectin-3 binding protein links circulating microparticles with electron dense glomerular deposits in lupus nephritis. Lupus 2015; 24: $1150-60$.

20. Larsen L, Chen HY, Saegusa J, Liu FT. Galectin-3 and the skin. J Dermatol Sci 2011; 64: 85-91.
21. Shi ZR, Tan GZ, Meng Z, et al. Association of anti-acidic ribosomal protein $\mathrm{PO}$ and anti-galectin 3 antibodies with the development of skin lesions in systemic lupus erythematosus. Arthritis Rheumatol 2015; 67: 193-203.

22. Taniguchi T, Asano Y, Akamata K, et al. Serum levels of galectin-3: possible association with fibrosis, aberrant angiogenesis, and immune activation in patients with systemic sclerosis. J Rheumatol 2012; 39: 539-44. 